doi:10.13108/2020-12-4-90

\title{
ON NECESSARY AND SUFFICIENT CONDITION IN THEORY OF REGULARIZED TRACES
}

\author{
Z.YU. FAZULLIN, N.F. ABUZYAROVA
}

\begin{abstract}
The present work is devoted to studying the regularized trace formulae for symmetric $L_{0}$-compact perturbations of a self-adjoint lower semi-bounded operator $L_{0}$ with a discrete spectrum in a separable Hilbert space. By now, the studies of the regularized trace formulae for the perturbations of abstract self-adjoint discrete operators were mostly aimed on finding a sufficient condition, under which the regularized sum with brackets minus first or several leading terms of the perturbation theory vanished. This condition was formulated in terms of spectral characteristics of an unperturbed operator $L_{0}$ depending on the belonging of a perturbing operator $V$ to some class. In particular, recently, the traces formulae for model two-dimensional operators in mathematical physics have been intensively studied with a perturbation described by the multiplication operator. Here we study a necessary and sufficient condition for two cases, namely, as the regularized trace with brackets and deduction of the first corrector of the perturbation theory vanishes or is equal to a finite number. We consider a certain summation bracket, which usually arises in the theory of regularized traces of the perturbations of partial differential operators.
\end{abstract}

Keywords: trace of an operator, resolvent, trace formula, perturbation theory, discrete spectrum.

Mathematics Subject Classification: 47A55, 47B02, 47B10, 47A10

\section{INTRODUCTION}

The theory of regularized traces theory for discrete operators was initiated by I.M. Gelfand and B.M. Levitan in work [1], where they considered a Sturm-Liouville operator with a potential $V(x)$ on a segment $[0, \pi]$ subject to the Dirichlet condition and they obtained a formula

$$
\sum_{k=1}^{\infty}\left(\mu_{k}-k^{2}-c_{0}\right)=-\frac{V(0)+V(\pi)}{4}+\frac{c_{0}}{2},
$$

with

$$
c_{0}=\pi^{-1} \int_{0}^{\pi} V(x) d x .
$$

Later, this formula was called Gelfand-Levitan formula.

Z.Yu. Fazullin, N.F. Abuzyarova, On the neCessary and Sufficient CONDition in the theory OF REGULARIZED TRACES.

(c) Fazullin Z.Yu., Abuzyarova N.F. 2020.

The research is made in the framework of state task of the Ministry of Science and Higher Education of Russian Federation (code of scientific theme FZWU-2020-0027) (Abuzyarova N.F.) and in the framework of the development program of Scientific and Educational Mathematical Center of Privolzhsky Federal District, additional agreement no. 075-02-2020-1421/1 to agreement no. 075-02-2020-1421 (Fazullin Z.Yu.).

Submitted August 21, 2020. 
In formula (1.1), the symbols $\mu_{k}$ denote the eigenvalues of the Sturm-Liouville operator and $k^{2}=\lambda_{k}$ are the eigenvalues of the same problem as $V(x) \equiv 0$.

Right after this work, L.A. Dikii showed in [2] that formula (1.1) is equivalent to the following identity:

$$
\sum_{k=1}^{\infty}\left(\mu_{k}-\lambda_{k}-\left(V f_{k}, f_{k}\right)\right)=0
$$

where $f_{k}(x)=\sqrt{\frac{2}{\pi}} \sin k x$ are the orthonormalized eigenfunctions of the Sturm-Liouville operator as $V(x) \equiv 0$ subject to the Dirichlet condition.

Namely, since

$$
\left(V f_{k}, f_{k}\right)=\frac{1}{\pi} \int_{0}^{\pi} V(x) d x-\frac{1}{\pi} \int_{0}^{\pi} V(x) \cos 2 k x d x,
$$

we can keep the diverging terms in the left hand side of identity 1.2 and summing up the other terms, we arrive at (1.1).

Exactly such approach, when the term $\left(V f_{k}, f_{k}\right)$, the first corrector of the perturbation theory, is to be studied, a diverging part is extracted and the rest is summed up, was main in numerous studies of the traces for perturbations of both regular and singular ordinary differential operators. A rather detailed survey was provided in work [3]. At the same time, the authors rather successfully developed direct methods for obtaining trace formulae like (1.1) skipping identity 1.2 .

From the end of $70 \mathrm{~s}$, the study of the traces of perturbations of partial differential operators became the primary topic. However, because of a complicated structure of the first corrector $\left(V f_{k}, f_{k}\right)$, it can not be always effectively studied and the same concerns further correctors. As one of the difficulties, we can mention the fact that the multiplicities $\nu_{k}$ of the eigenvalues $\lambda_{k}$ increases unboundedly as $k \rightarrow \infty$ and the spectral gaps do not grow. This is why direct methods for obtaining trace formulae for the perturbations of partial differential operators based on asymptotic formulae and representations for the eigenvalues $\mu_{k}$ of perturbed ordinary differential operators do not work. In view of this, active studies of formulae like 1.2 and close ones with deducting several correctors of the perturbation theory have been initiated for a general case, namely, for the perturbations of abstract discrete self-adjoint operators.

Let $L_{0}$ be a self-adjoint lower-semibounded operator in a separable Hilbert space $H$ with a pure discrete spectrum, $\left\{\lambda_{k}\right\}_{k=1}^{\infty}$ be the eigenvalues of the operator $L_{0}$ taken in the ascending order with the multiplicities taken into account, $\lambda_{k} \leqslant \lambda_{k+1}, k=1,2, \ldots$, and $\left\{f_{k}\right\}_{k=1}^{\infty}$ be the orthonormalized basis formed by the eigenfunctions of the operator $L_{0}$. Let $V$ be a symmetric $L_{0}$-compact operator in $H$, then by the well-known Kato-Rellich theorem, the operator $L=$ $L_{0}+V$ is closed on the domain of the operator $L_{0}$ and has a pure discrete spectrum. Let $\left\{\mu_{k}\right\}_{k=1}^{\infty}$ be the eigenvalues of the operator $L$ taken in the ascending order with the multiplicities taken into account $\mu_{k} \leqslant \mu_{k+1}, k=1,2, \ldots$

We note that since both the operators $L_{0}$ and $L=L_{0}+V$ are lower-semibounded, without loss of generality we can suppose that

$$
\lambda_{k} \geqslant \delta>0, \quad \mu_{k} \geqslant \delta>0
$$

for each $k$.

For the described general self-adjoint operators with a pure discrete spectrum we formulate a following problem. 
What conditions for the operators $L_{0}$ and $V$ do ensure the existence of a subsequence $\left\{n_{m}\right\}_{m=1}^{\infty}$ of natural numbers such that

$$
\lim _{m \rightarrow \infty} \sum_{k=1}^{n_{m}}\left[\lambda_{k}+\left(V f_{k}, f_{k}\right)-\mu_{k}\right]=0
$$

Identity $(1.3)$ is called a regularized trace formula with brackets and deducting the first corrector of the perturbation theory; it generalizes identity $(1.2)$ for abstract discrete operators.

For such general problem on validity of identity (1.3), a pioneering work was written by V.A. Sadovnichii, V.V. Dubrovsky, V.A. Lubishkin [4]. Let us mention the most valuable successes in this direction.

In work [5], V.A. Sadovnichii and V.E. Podolskii proved identity (1.3) for arbitrary bounded perturbations $V$ being a Hilbert-Schmidt operator if $R_{0}(z)=\left(L_{0}-z I\right)^{-1}$ is a nuclear operator and the spectrum of the operator $L_{0}$ possesses growing gaps, that is, if there exist a subsequence $\left\{\lambda_{n_{m}}\right\}_{m=1}^{\infty}$ such that $\lambda_{n_{m+1}}-\lambda_{n_{m}} \rightarrow \infty$ as $m \rightarrow \infty$. We note that a core of the proof in this work and previous numerous works by V.A. Sadovnichii, V.V. Dubrovsky, V.A. Lubishkin and other authors was based on applying the operator $\operatorname{tr}\left(-\frac{1}{2 \pi} \oint_{\Gamma_{m}} z(\cdot) d z\right)$ to the resolvent identity

$$
R(z)=R_{0}(z)-R_{0}(z) V R_{0}(z)+\left(R_{0}(z) V\right)^{2} R(z)
$$

where

$$
\Gamma_{m}=\left\{z:|z|=2^{-1}\left(\lambda_{n_{m}}+\lambda_{n_{m+1}}\right)\right\}, \quad R(z)=(L-z I)^{-1} .
$$

A next essential advantage in this direction was made in a work by Kh.Kh. Murtazin and Z.Yu. Fazullin [6]. In order to describe the results and ideas from this work concerning identity (1.3), we introduce a point-wise indexation of the operator $L_{0}$ :

$$
\sigma\left(L_{0}\right)=\left\{\bar{\lambda}_{k}\right\}_{k=1}^{\infty}, \quad \bar{\lambda}_{k}<\bar{\lambda}_{k+1}, \quad k=1,2, \ldots
$$

We denote by $P_{k}$ the orthogonal projector on the eigenspace of the operator $L_{0}$ associated with the eigenvalue $\bar{\lambda}_{k}$ and let $\nu_{k}=\operatorname{dim} \operatorname{Ran} P_{k}$ be the multiplicity of the eigenvalue $\bar{\lambda}_{k}$ so that

$$
P_{k}(\cdot)=\sum_{i=1}^{\nu_{k}}\left(\cdot, f_{k_{i}}\right) f_{k_{i}}
$$

where $\left\{f_{k_{i}}\right\}_{i=1}^{\nu_{k}}$ is a basis formed by the eigenfunctions of the operator $L_{0}$ in the subspace $P_{k} H$. Let $\mu_{i}^{(k)}, i=\overline{1, \nu_{k}}$ be a group of the eigenvalues of the operator $L$ arising after the splitting of an eigenvalue $\bar{\lambda}_{k}$ of the operator $L_{0}$ under the perturbation by the operator $V$; these eigenvalues are taken in the ascending order counting their multiplicities.

Hereafter, the following condition is assumed: the operator $K_{0}(\lambda)=\left(R_{0}(-\lambda) V\right)^{2} R_{0}(-\lambda)$, $\lambda>0$, is nuclear, that is,

$$
\operatorname{tr} K_{0}(\lambda)=\sum_{k=1}^{\infty} \sum_{m=1}^{\infty} \frac{a_{m k}}{\left(\bar{\lambda}_{k}+\lambda\right)^{2}\left(\bar{\lambda}_{m}+\lambda\right)}<\infty,
$$

where $a_{m k}=\operatorname{tr} P_{k} V P_{m} V$.

The following results and ideas were presented in work [6]. First, the following identity was one of the key ingredients:

$$
0 \leqslant \rho(t) \stackrel{\text { def }}{=} \sum_{\lambda_{k}<t}\left[\lambda_{k}+\left(V f_{k}, f_{k}\right)-\mu_{k}\right]=\sum_{\bar{\lambda}_{k}<t}\left[\sum_{i=1}^{\nu_{k}}\left(\bar{\lambda}_{k}-\mu_{i}^{(k)}\right)+\operatorname{tr} P_{k} V\right]
$$


for each $t \in K$, where $K$ is a compact set in $\mathbb{R}$, see [6, Lm. 1.4]. Then for symmetric $L_{0}$-compact perturbations $V$, i.e.,

$$
\left\|R_{0}(-\lambda) V\right\| \rightarrow 0 \quad \text { as } \quad \lambda \rightarrow+\infty
$$

the following asymptotic identity was proved [6, Thm. 1.3]:

$$
\int_{0}^{\infty} \frac{\omega(t) d t}{(t+\lambda)^{4}}=\int_{0}^{\infty} \frac{\nu(t) d t}{(t+\lambda)^{4}}\left[1+O\left(\left\|R_{0}(-\lambda) V\right\|\right)\right]
$$

where

$$
\begin{aligned}
& \omega(t)=2 \int_{0}^{t} \rho(\tau) d \tau+\sum_{\lambda_{k}<t}\left(\lambda_{k}-\mu_{k}\right)^{2}, \\
& \nu(t)=2 \int_{0}^{t} \tau(s) d s+\sum_{\bar{\lambda}_{k}<t} a_{k k}, \\
& \tau(s)=\sum_{\bar{\lambda}_{k}<s} \sum_{\bar{\lambda}_{m} \geqslant s} a_{m k}\left(\bar{\lambda}_{m}-\bar{\lambda}_{k}\right)^{-1} .
\end{aligned}
$$

We also mention that if $K_{0}(\lambda)$ is a nuclear operator, then [6, Eq. (1.47)]

$$
f(\lambda) \stackrel{\text { def }}{=} \operatorname{tr} K_{0}(\lambda)=3 \int_{0}^{\infty} \frac{\nu(t)}{(t+\lambda)^{4}} d t .
$$

Employing identities (1.6) and (1.7), it was proved [6, Lm. 1.10] that if

$$
f(\lambda)=o\left(\lambda^{-2}\right) \text { for } \lambda \gg 1,
$$

then there exists a subsequence $\left\{n_{m}\right\}_{m=1}^{\infty} \subseteq \mathbb{N}$ such that identity 1.3 holds true. It should be stressed that condition $(1.8)$ is not related with any particular class of the perturbations $V$.

On the base of this statement, namely, on the base of Lemma 1.10 in [6], there were obtained sufficient conditions ensuring identity $(1.3)$ for various classes of perturbations $V$. In order to provide a complete picture, we reproduce here Theorem 1.5 from [6].

Theorem A. Let $L_{0}=L_{0}^{*}$ be a lower semi-bounded operator with a discrete spectrum and $V$ be a symmetric $L_{0^{-}}$compact operator in $H$. Assume that one of the following conditions hold:

(1) $\sum_{k=1}^{\infty} \frac{\left(V^{2} f_{k}, f_{k}\right)}{\lambda_{k}}<\infty$;

(2) $V$ is bounded and $N(t)=\bar{o}(t)$ as $t \rightarrow \infty$;

(3) $V$ is compact and $N(t)=O(t)$ as $t \rightarrow \infty$;

(4) $V \in \sigma_{p}, 2<p \in \mathbb{N}$ and $N(\bar{t})=\bar{o}\left(t^{\frac{p}{p-2}}\right)$ as $t \rightarrow \infty$;

(5) $V \in \sigma_{2}$.

Then there exists a subsequence of natural numbers $\left\{n_{m}\right\}_{m=1}^{\infty}$ such that

$$
\lim _{m \rightarrow \infty} \sum_{k=1}^{n_{m}}\left(\lambda_{k}+\left(V f_{k}, f_{k}\right)-\mu_{k}\right)=0 .
$$

In this theorem, the symbol $\sigma_{p}, p \in \mathbb{N}$, denotes the Schatten-von Neumann class of compact operators and in particular, $\sigma_{2}$ is the class of Hilbert-Schmidt operators. We should say that Theorem A covers all previously known results in the case of symmetric $L_{0}$-compact operators $V$. 
The present work is the first devoted to studying a necessary and sufficient condition guaranteeing that the sum of the series is equal either to zero or to a finite non-zero number, depending on the way of placing the brackets. Namely, we consider the series

$$
\sum_{k=1}^{\infty} a_{k}=\sum_{k=1}^{\infty}\left[\sum_{i=1}^{\nu_{k}}\left(\bar{\lambda}_{k}-\mu_{i}^{(k)}\right)+\operatorname{tr} P_{k} V\right] .
$$

Such placing of brackets usually arises in studying, for instance, the trace formulae for perturbations of model partial differential operators in mathematical physics, see works [7], [9], [6], [11, [12], [13].

\section{CRiterion For VAlue of Regularized trace}

We first transform identity (1.6). First we note that if the operator is $L_{0}$-compact and condition (1.4) is satisfied, then by Theorem 1.2 in work [6] we obtain that

$$
2 \int_{0}^{\infty} \frac{\rho(t) d t}{(t+\lambda)^{3}}<\operatorname{tr} K_{0}(\lambda)<\infty
$$

The following lemma holds.

Lemma 1. Let the operator $K_{0}(\lambda)$ be nuclear and $V$ be symmetric and $L_{0}$-compact. Then

$$
\sum_{k=1}^{\infty} \frac{a_{k}}{\left(\bar{\lambda}_{k}+\lambda\right)^{2}}=2 \int_{0}^{\infty} \frac{\rho(t) d t}{(t+\lambda)^{3}} .
$$

Proof. Since $\rho(t) \geqslant 0$, the definition of $\rho(t)$ implies that for each $n \in \mathbb{N}$ we have

$$
\sum_{k=1}^{n} \frac{a_{k}}{\left(\lambda_{k}+\lambda\right)^{2}}=\int_{0}^{\bar{\lambda}_{n}} \frac{d \rho(t)}{(t+\lambda)^{2}}=\frac{\rho\left(\bar{\lambda}_{n}+0\right)}{\left(\bar{\lambda}_{n}+\lambda\right)^{2}}+2 \int_{0}^{\bar{\lambda}_{n}} \frac{\rho(t)}{(t+\lambda)^{3}} d t .
$$

Identity 2.2 will be implied by 2.3 once we show that

$$
\lim _{n \rightarrow \infty} \frac{\rho\left(\bar{\lambda}_{n}+0\right)}{\left(\bar{\lambda}_{n}+\lambda\right)^{2}}=0 .
$$

We prove the above identity arguing by contradiction; in view of the inequality $\rho(t) \geqslant 0$ we suppose that

$$
\lim _{n \rightarrow \infty} \frac{\rho\left(\bar{\lambda}_{n}+0\right)}{\left(\bar{\lambda}_{n}+\lambda\right)^{2}}=a>0
$$

Then

$$
\rho\left(\bar{\lambda}_{n}+0\right) \geqslant(a-\varepsilon)\left(\bar{\lambda}_{n}+\lambda\right)^{2}, \quad \varepsilon=\frac{a}{2},
$$

for all $n \geqslant n_{0}(\varepsilon)$. Since the function $\rho_{n}(t)$ is non-negative and step-like, we get:

$$
\lim _{r \rightarrow \infty} \int_{0}^{r} \frac{\rho(t)}{(t+\lambda)^{3}} d t=+\infty
$$

which contradicts inequality 2.1. The proof is complete. 
Integrating by parts, we confirm that

$$
2 \int_{0}^{+\infty} \frac{\rho(t)}{(t+\lambda)^{3}} d t=3 \int_{0}^{\infty} \frac{2 \int_{0}^{t} \rho(\tau) d \tau}{(t+\lambda)^{4}} d t
$$

Now identities (1.6), (2.2), (2.4) and Lemma 1.7 in work [6] yield that for $\lambda \gg 1$, the identity

$$
\sum_{k=1}^{\infty} \frac{a_{k}}{\left(\bar{\lambda}_{k}+\lambda\right)^{2}}=f_{1}(\lambda)\left[1+O\left(\left\|R_{0}(-\lambda) V\right\|\right)\right]
$$

holds, where

$$
f_{1}(\lambda)=3 \int_{0}^{+\infty} \frac{\int_{0}^{t} \tau(s) d s+\sum_{\bar{\lambda}_{k}<t}\left[a_{k k}-\sum_{i=1}^{\nu_{k}}\left(\bar{\lambda}_{k}-\mu_{i}^{(k)}\right)^{2}\right]}{(t+\lambda)^{4}} d t
$$

Theorem 1. Assume that series (1.9) converges. Then the sum of series (1.9) vanishes if and only if

$$
f_{1}(\lambda)=\bar{o}\left(\lambda^{-2}\right) \quad \text { as } \quad \lambda \rightarrow+\infty .
$$

Proof. Necessity. Let $\sum_{k=1}^{\infty} a_{k}=0$. We are going to show that the series

$$
\sum_{k=1}^{\infty} \frac{\lambda^{2}}{\left(\bar{\lambda}_{k}+\lambda\right)^{2}} a_{k}
$$

converges uniformly for all $\lambda>0$. Indeed, since the functional sequence

$$
b_{k}(\lambda)=\frac{\lambda^{2}}{\left(\bar{\lambda}_{k}+\lambda\right)^{2}}
$$

is monotone and is uniformly bounded on the set $\lambda>0$ and the series $\sum_{k=1}^{\infty} a_{k}$ converges, then the considered series converges uniformly on the set $\lambda>0$ by the Abel test of uniform convergence. Therefore,

$$
\lim _{\lambda \rightarrow+\infty} \lambda^{2} \sum_{k=1}^{\infty} \frac{a_{k}}{\left(\bar{\lambda}_{k}+\lambda\right)^{2}}=\sum_{k=1}^{\infty} \lim _{\lambda \rightarrow+\infty} \frac{\lambda^{2}}{\left(\bar{\lambda}_{k}+\lambda\right)^{2}} a_{k}=\sum_{k=1}^{\infty} a_{k}=0 .
$$

Since the operator $V$ is $L_{0}$-compact, that is,

$$
\left\|R_{0}(-\lambda) V\right\| \rightarrow 0 \quad \text { as } \quad \lambda \rightarrow+\infty
$$

identities (2.5) and (2.7) imply relation (2.6).

Sufficiency. Assume that condition 2.6 holds. As we have mentioned above,

$$
\left\|R_{0}(-\lambda) V\right\| \rightarrow 0 \quad \text { as } \quad \lambda \rightarrow+\infty,
$$

and hence, it follows from identity 2.5 that

$$
\sum_{k=1}^{\infty} \frac{a_{k}}{\left(\bar{\lambda}_{k}+\lambda\right)^{2}}=o\left(\lambda^{-2}\right)
$$

for $\lambda \gg 1$. 
Suppose the opposite, that is, $\sum_{k=1}^{\infty} a_{k}=c_{0}>0$; the constant $c_{0}$ is strictly positive by inequality 1.5p). Since the series $\sum_{k=1}^{\infty} \frac{\lambda^{2}}{\left(\lambda_{k}+\lambda\right)^{2}} a_{k}$ converges uniformly for all $\lambda>0$, we have:

$$
\lim _{\lambda \rightarrow+\infty} \lambda^{2} \sum_{k=1}^{\infty} \frac{a_{k}}{\left(\bar{\lambda}_{k}+\lambda\right)^{2}}=\sum_{k=1}^{\infty} \lim _{\lambda \rightarrow+\infty} \frac{\lambda^{2}}{\left(\bar{\lambda}_{k}+\lambda\right)^{2}} a_{k}=\sum_{k=1}^{\infty} a_{k}=c_{0} .
$$

This implies identity

$$
\sum_{k=1}^{\infty} \frac{a_{k}}{\left(\bar{\lambda}_{k}+\lambda\right)^{2}}=O\left(\frac{1}{\lambda^{2}}\right), \quad \lambda \rightarrow+\infty,
$$

which contradicts (2.8). The proof is complete.

Theorem 2. Let series (1.9) converge. Then relation

$$
\sum_{k=1}^{\infty} a_{k}=c_{0}>0
$$

holds if and only if

$$
f_{1}(\lambda) \sim c_{0} \lambda^{-2} \quad \text { as } \quad \lambda \rightarrow+\infty
$$

Proof. Necessity. Assume that 2.10 holds. Since the operator $V$ is $L_{0}$-compact, we have

$$
\left\|R_{0}(-\lambda) V\right\| \rightarrow 0 \quad \text { as } \quad \lambda \rightarrow+\infty .
$$

Therefore,

$$
f_{1}(\lambda)\left[1+\left\|V R_{0}(-\lambda)\right\|\right] \sim f_{1}(\lambda) \quad \text { as } \quad \lambda \rightarrow+\infty .
$$

We multiply identity 2.5 by $\lambda^{2} \cdot c_{0}^{-1}$ and in the obtained identity we pass to the limit as $\lambda \rightarrow+\infty$. According identity 2.9 we then get that

$$
1=\lim _{\lambda \rightarrow+\infty} \frac{f_{1}(\lambda)\left[1+\left\|V R_{0}(-\lambda)\right\|\right]}{c_{0} \cdot \lambda^{-2}},
$$

that is,

$$
\frac{c_{0}}{\lambda^{2}} \sim f_{1}(\lambda)\left[1+\left\|V R_{0}(-\lambda)\right\|\right]
$$

In view of relation 2.12, this implies 2.11.

Sufficiency. Let

$$
f_{1}(\lambda) \sim c_{0} \lambda^{-2} \text { as } \lambda \rightarrow+\infty,
$$

where $c_{0}$ is a positive constant. Multiplying identity 2.5 by $\lambda^{2}$, we get

$$
\sum_{k=1}^{\infty} \frac{\lambda^{2}}{\left(\bar{\lambda}_{k}+\lambda\right)^{2}} a_{k}=\lambda^{2} f_{1}(\lambda)\left[1+O\left\|R_{0}(-\lambda) V\right\|\right]
$$

We recall the aforementioned uniform convergence for $\lambda>0$ of the series in the left hand side in 2.13). Taking into consideration this fact and the equivalence of (2.11) and (2.12), we pass to the limit as $\lambda \rightarrow+\infty$ in 2.13 . Then we find that

$$
\sum_{k=1}^{\infty} a_{k}=c_{0}
$$

The proof is complete. 
Later we are going to establish the validity of estimates (2.6) and (2.11) for the function $f_{1}(\lambda)$ for particular classes of perturbations $V$. Since at that we do not assume the existence of growing gaps in the spectrum of the operator $L_{0}$ (this is related with a particular choice of summation brackets), we shall have to impose a condition for the eigenvalues $\mu_{i}^{(k)}, i=\overline{1, \nu_{k}}$ of the operator $L$ : as $k \gg 1$, the eigenvalues $\mu_{i}^{(k)}$ are located in the vicinity of the eigenvalue $\bar{\lambda}_{k}$, that is, $\left|\mu_{i}^{(k)}-\bar{\lambda}_{k}\right|<r_{k}, i=\overline{1, \nu_{k}}$, where

$$
r_{k}=\min \left\{\left(\bar{\lambda}_{k}-\bar{\lambda}_{k-1}\right) \cdot 2^{-1},\left(\bar{\lambda}_{k+1}-\bar{\lambda}_{k}\right) \cdot 2^{-1}\right\} .
$$

Let

$$
R_{0 k}(z)=R_{0}(z)-\left(z-\bar{\lambda}_{k}\right)^{-1} P_{k}
$$

be the reduced resolvent of the operator $L_{0}$ in the vicinity of the eigenvalue $\bar{\lambda}_{k}$. Then, if

$$
\lim _{k \rightarrow \infty} \sup _{\left|z-\bar{\lambda}_{k}\right| \leqslant r_{k}}\left\|R_{0 k}(z) V\right\|=0
$$

then it is easy to show that

$$
\gamma(t):=\sum_{\bar{\lambda}_{k}<t}\left[a_{k k}-\sum_{i=1}^{\nu_{k}}\left(\bar{\lambda}_{k}-\mu_{i}^{(k)}\right)^{2}\right]=\vec{o}(t)
$$

as $t \gg 1$. Therefore,

$$
f_{1}^{(2)}(\lambda)=3 \int_{0}^{\infty} \frac{\gamma(t)}{(t+\lambda)^{4}} d t=o\left(\lambda^{-2}\right), \quad \lambda \rightarrow+\infty .
$$

This is why the validity of estimates (2.6) and (2.11) for various classes of perturbations $V$ should be established for the function

$$
f_{1}^{(1)}(\lambda)=3 \int_{0}^{\infty} \frac{2 \int_{0}^{t} \tau(s) d s}{(t+\lambda)^{4}} d t
$$

since

$$
f_{1}(\lambda)=f_{1}^{(1)}(\lambda)+f_{1}^{(2)}(\lambda)
$$

In conclusion we formulate one more problem:

Prove that there exists a subsequence of natural numbers $\left\{n_{m}\right\}_{m=1}^{\infty}$ such that

$$
\lim _{m \rightarrow \infty} \sum_{k=1}^{n_{m}}\left[\lambda_{k}+\left(V f_{k}, f_{k}\right)-\mu_{k}\right]=0
$$

if and only if

$$
f_{1}(\lambda)=o\left(\lambda^{-2}\right) \quad \text { as } \quad \lambda \rightarrow+\infty .
$$

We mention that the proof of the sufficiency is not difficult and is clear for us. The justification of the necessity is not completed yet and requires further studies.

\section{BIBLIOGRAPHY}

1. I.M. Gelfand, B.M. Levitan. On a simple identity for eigenvalues of second differential oeprator // Doklady AN SSSR. 88, 593-596 (1953). (in Russian).

2. L.A. Dikii. On a formula of Gelfand-Levitan // Uspekhi Matem. Nauk. 8:2 119-123 (1953). (in Russian).

3. V.A. Sadovnichii, V.E. Podolskii. Traces of operators // Uspekhi Matem. Nauk. 61:5, 89-156 (2006). [Russ. Math. Surveys. 61:5, 885-953 (2006).] 
4. V.A. Sadovnichij, V.V. Dubrovskij, V.A. Lyubishkin. Traces of discrete operators // Dokl. AN SSSR. 264:4, 830-832 (1982). [Sov. Math. Dokl. 25, 759-761 (1982).]

5. V.A. Sadovnichii, V.E. Podolskii. Traces of operators with relatively compact perturbations // Matem. Sborn. 193:2, 129-152 (2002). [Sb. Math. 193:2, 279-302 (2002).

6. Kh.Kh. Murtazin, Z.Yu. Fazullin. Non-nuclear perturbations of discrete operators and trace formulae // Matem. Sborn. 196:12, 123-156 (2005). [Sb. Math. 196:12, 1841-1874 (2005).]

7. V.A. Sadovnichii and V. V. Dubrovskii. A classical regularized trace formula for the eigenvalues of the Laplace-Beltrami operator with a potential on the sphere $S^{2} / /$ Dokl. Akad. Nauk SSSR. 319:1, 61-62 (1991). [Soviet Math. Dokl. 44:1, 56-58 (1992).]

8. V.A. Sadovnichij, Z.Yu. Fazullin. A formula for the first regularized trace of a perturbed LaplaceBeltrami operator // Diff. Uravn. 37:3, 402-409 (2001). [Diff. Equat. 37:3, 430-438 (2001).]

9. Z.Yu. Fazullin, Kh.Kh. Murtazin. Regularized trace of a two-dimensional harmonic oscillator // Matem. Sborn. 192:5, 87-124 (2001). [Sb. Math. 192:5, 725-761 (2001).]

10. Kh.Kh. Murtazin, F.Yu. Fazullin. The spectrum and trace formula for the two-dimensional Schrödinger operator in a homogeneous magnetic field// Dokl. Akad. Nauk. 390:6, 743-745 (2003). [Dokl. Math. 67:3, 426-428 (2003).]

11. E. Korotyaev, A. Pushniski. A trace formula and high-energy spectral asymptotics for the perturbed Landau hamiltonian // J. Funct. Anal. 217:1, 221-248 (2004).

12. A.I. Atnagulov, V.A. Sadovnichii, Z.Yu. Fazullin. Properties of the resolvent of the Laplace operator on a two-dimensional sphere and a trace formula // Ufimskij Matem. Zhurn. 8:3, 22-40 (2016). [Ufa Math. J. 8:3, 22-40 (2016).]

13. Z.Yu. Fazullin, I.G. Nugaeva. Spectrum and a trace formula for a compactly supported perturbation of the 2D harmonic oscillator in a strip // Differ. Uravn. 55:5, 691-701 (2019). [Diff. Equat. 55:5, 677-687 (2019).]

Ziganur Yusupovich Fazullin, Bashkir State University,

Zaki Validi str. 32,

450076, Ufa, Russia

E-mail: fazullinzu@mail.ru

Natalia Fairbakhovna Abuzyarova,

Bashkir State University,

Zaki Validi str. 32,

450076, Ufa, Russia

E-mail: abnatf@gmail.com 